\title{
RELACIÓN ENTRE ASIMETRÍA FLUCTUANTE Y EL TRATAMIENTO HORMONAL, CIRUGÍA-ORTODONCIA MAXILOFACIAL, TRAUMATISMOS Y MALFORMACIONES CRANEOFACIALES
}

\author{
EFFECTS OF HORMONAL TREATMENT, MAXILOFACIAL SURGERY- \\ ORTHODONTICS, TRAUMATISM, AND MALFORMATION ON \\ FLUCTUATING ASYMMETRY
}

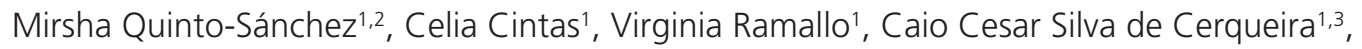
Jorge Gomez-Valdés ${ }^{4}$, Victor Acuña-Alonzo 5,6 , Kaustubh Adhikari ${ }^{5}$, Paola Everardo ${ }^{6}$, Francisco de Avila ${ }^{6}$, Carla Jaramillo7, Williams Arias ${ }^{7}$, Macarena Fuentes ${ }^{5,8}$, Tábita Hünemeier ${ }^{9}$, Carla Gallo $^{10}$, Giovani Poletti ${ }^{10}$, Javier Rosique7, Lavinia Schuler-Faccini ${ }^{9}$, Maria Catira Bortolini ${ }^{9}$, Samuel Canizales-Quinteros ${ }^{11}$, Francisco Rothhammer ${ }^{8}$, Gabriel Bedoya7 ${ }^{7}$ Andres Ruiz-Linares ${ }^{5}$ y Rolando Gonzalez-José ${ }^{*}$
\end{abstract}

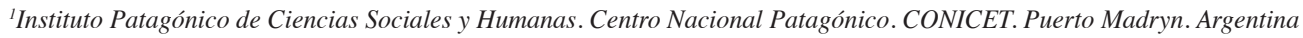
${ }^{2}$ Licenciatura en Ciencias Forenses. Facultad de Medicina. Universidad Nacional Autónoma de México (UNAM). Ciudad de México. México

${ }^{3}$ Policía Técnico-Científica del Estado de Sao Paulo. Ourinhos. Brasil

${ }^{4}$ Posgrado en Antropología Física. Escuela Nacional de Antropología e Historia. Ciudad de México. México

${ }^{5}$ Department of Genetics, Evolution and Environment, and UCL Genetics Institute. University College London. London. United Kingdom ${ }^{6}$ Escuela Nacional de Antropología e Historia. Instituto Nacional de Antropología e Historia. Ciudad de México. México ${ }^{7}$ Departamento de Antropología. Facultad de Ciencias Humanas y Sociales. Universidad de Antioquia. Medellín. Colombia ${ }^{8}$ Instituto de Alta Investigación Universidad de Tarapacá. Programa de Genética Humana ICBM. Facultad de Medicina Universidad de Chile y Centro de Investigaciones del Hombre en el Desierto. Arica. Chile

${ }^{9}$ Departamento de Genética. Instituto de Biociências. Universidade Federal do Rio Grande do Sul. Porto Alegre. Brasil

${ }^{10}$ Laboratorios de Investigación y Desarrollo. Facultad de Ciencias y Filosofía. Universidad Peruana Cayetano Heredia. Lima. Perú

${ }^{11}$ Unidad de Genómica de Poblaciones Aplicada a la Salud. Facultad de Química. UNAM-Instituto Nacional de Medicina Genómica. Ciudad de México. México
\end{abstract}

PALABRAS CLAVE asimetría fluctuante facial; inestabilidad del desarrollo; tratamiento hormonal; morfometría geométrica

RESUMEN En este trabajo se evalúa la relación entre la asimetría fluctuante facial (AFF) y los tratamientos hormonales, cirugías maxilofaciales, ortodoncia, traumatismos y malformaciones. En el marco del proyecto CANDELA, se tomaron cinco fotografías faciales de 3162 voluntarios entre los 18 y 85 años. Por fotogrametría se colocaron 34 landmarks o puntos en 3D y mediante el método Procrustes ANOVA se obtuvieron valores individuales de asimetría fluctuante facial. Se realizó una prueba de ANOVA de una vía y la prueba de Welch y Levene para conocer las diferencias entre media y varianza de los valores de asimetría facial y las variables respuesta. También, se caracterizó la variación morfológica del componente asimétrico de la forma facial mediante técnicas multivariadas sobre los grupos que resultaran diferentes significativamente. Las mujeres que reportaron haber recibido algún tipo de tratamiento hormonal mostraron mayores valores de asimetría fluctuante facial respecto al grupo sin tratamiento. Esta asociación se mantuvo una vez removido el efecto de la heterocigosidad (como indicador de la ancestría) y sin interactuar con el resto de covariables incluidas en el análisis. Los cambios morfológicos asociados a este factor se concentran en el mentón, maxilar labio inferior, región perifrontal, región nasal y orejas. Algunos trabajos anteriores dieron cuenta de la posible relación entre la asimetría facial y los niveles de hormonas, pero no hay estudios que sustenten la relación causal o directa entre la asociación aquí planteada. El presente trabajo es una evidencia más de la asociación entre el consumo de hormonas y modificaciones de caracteres faciales en poblaciones urbanas mestizas latinoamericanas. Rev Arg Antrop Biol 20(1), 2018. doi:10.17139/raab.2018.0020.01.06

Financiamiento: Programa de Becas Latinoamericanas CONICET (N41488 a M.Q-S). Leverhulme Trust (F/07 134/DF a A.R-L). BBSRC (BB/I021213/1 a A.R-L).

*Correspondencia a: Rolando Gonzalez-José. Bvd. Brown 2915. U9120ACD Puerto Madryn. Chubut Argentina. E-mail: rolando@cenpat-conicet.gob.ar

Recibido 24 Marzo 2016; aceptado 11 Septiembre 2017

doi:10.17139/raab.2018.0020.01.06 
KEY WORDS facial fluctuating asymmetry; development instability; hormonal treatment, geometric morphometrics

ABSTRACT In this work we test for the putative association between facial fluctuating asymmetry (FFA) and hormone treatments, maxillofacial surgery, orthodontics, injuries, and malformations. A protocol of five photographs and photogrammetric reconstruction was implemented to place thirty-four 3D landmarks in 3162 individuals aged between 18 and 85 years, belonging to the CANDELA initiative. A Procrustes ANOVA test was used to obtain individual facial fluctuating asymmetry scores. One way ANOVA, Welch, and Levene tests were conducted to explore the potential differences between mean and variance of the response variables. Our results indicate that women who received some hormonal treatment showed higher fluctuating facial asymmetry scores in relation to the unaffected group, this being persistent once the effects of heterozygosity (genetic ancestry) and further variables had been statistically controlled. The shape changes corresponding to this association are focused on the chin, jaw, lower lip, prefrontal region, nose, and ears. Previous reports suggested a potential relationship between facial asymmetry and hormone levels, but to the best of our knowledge there are no reports indicating the causation underlying the association detected here. This report is one more evidence of the association between hormone intake and facial asymmetric features in urban admixed Latin American populations. Rev Arg Antrop Biol 20(1), 2018. doi:10.17139/raab.2018.0020.01.06
La asimetría fluctuante es un carácter cuantitativo analizado en rasgos fenotípicos bilaterales corporales que es considerado indicador universal de inestabilidad en el desarrollo, tanto en humanos como en otras especies (Palmer y Strobeck, 1986; Leary y Allendorf, 1989; Clarke, 1993; Møller, 1990, 1996; Møller y Swaddle, 1997; Allenbach et al., 1999; Milne et al., 2003; Lens y van Dongen, 2008; Little et al., 2008, 2012; DeLeon y Richtsmeier, 2009; Özener, 2010a; Özener y Fink, 2010; Weisensee, 2013; Farrera et al., 2014; Quinto-Sánchez et al., 2015). La asimetría fluctuante es usada como marcador potencial de lo que se han denominado "buen componente genético" o fitness individual (Leamy y Klingenberg, 2005), a pesar de que la evidencia de una relación entre la asimetría y el fitness parece no ser tan precisa (McKenzie y Clarke, 1988). La hipótesis central es que niveles altos de asimetría fluctuante suelen coincidir con niveles igualmente altos de anomalías o "ruido" en el desarrollo. Sin embargo, la relación lineal entre asimetría e inestabilidad en el desarrollo ha sido cuestionada por algunos autores (McKenzie y Clarke, 1988; Graham et al., 1993; Bjorksten et al., 2000; Lens et al., 2002; Pound et al., 2014).

La asimetría fluctuante facial (AFF) ha sido estudiada en relación a un sinnúmero de hipótesis o variables respuesta, por ejemplo, comparando los niveles de asimetría en poblaciones rurales y urbanas (Gray y Marlowe, 2009), caracterizando las diferencias asociadas al estatus socioeconómico (Özener, 2010b, 2011), las condiciones de trabajo (Özener, 2010a), el dimorfismo sexual (Claes et al., 2012), la relación con la heterocigocidad y ancestría genética en mestizos (Quinto-Sánchez et al., 2015), comparando su patrón en las dismorfologías craneofaciales (DeLeon y Richtsmeier, 2009), explorando su importancia en el diagnóstico del síndrome alcohólico fetal (SAF) (Klingenberg et al., 2010b), caracterizando su distribución en algunos desórdenes neurológicos o síndromes (Burton et al., 2002), su relación con el ciclo menstrual (Manning et al., 1996), la atracción sexual (Farrera, 2011; Farrera et al., 2014) e incluso, dentro de la psicología evolucionista, analizando la relación con el estrés psicológico (Shackelford y Larsen, 1997). La caracterización de niveles de AFF base en poblaciones "naturales" y "sanas" también ha sido un tópico recurrente en trabajos de variación facial (Debat y David, 2001; Ercan et al., 2008; Farrera et al., 2014; Quinto-Sánchez et al., 2015), siempre concluyendo que a nivel poblacional existen valores basales de asimetría facial (Debat y David, 2001; Ercan et al., 2008; Farrera et al., 2014). De esta manera, en este documento entendemos la AFF como una respuesta multidimensional de la norma de reacción del desarrollo (Schlichting y Pigliucci, 1998) a variación ambiental interna (células y tejidos) o externa (ambiente, crecimiento, etc.).

En este contexto, existen dos propuestas sobre la etiología de la asimetría fluctuante: 1) que tiene su origen azaroso y no genético en las desviaciones de la capacidad individual para corregir la inestabilidad (Palmer et al., 1994) y en contraposición, 2) que es producto de los movimientos estocásticos de moléculas o células durante el desarrollo (Soulé, 1982; Emlen et al., 1993). Dentro de toda la gama de posibles variables respuesta a la asimetría tenemos, según Bishara et al., (2009) a) genéticas o malforma- 
ciones congénitas, por ejemplo, microsomía hemifacial y fisuras unilaterales de labio y paladar; b) factores ambientales, por ejemplo, hábitos de vida y traumatismos y c) funcionales, por ejemplo, cambios mandibulares como resultado de interferencias dentales, etc.

Existen caracterizaciones del patrón de asimetría y las malformaciones genéticas (DeLeon y Richtsmeier, 2009; Starbuck et al., 2013), funcionales, como el estudio de las maloclusiones (Sheats et al., 1998; Shah et al., 2009 o aquellos relacionados con factores ambientales como el consumo de alcohol durante la gestación (Klingenberg et al., 2010b), traumatismos faciales (Markey et al., 1980; Rajendra et al., 2009) o por el consumo de medicamentos (Enlow, 1990, 1996; Kasperk et al., 1997; Guendelman et al., 2000; Smith et al., 2006; Neave, 2008). Todas los anteriores son posibles escenarios de respuesta al patrón de asimetría fluctuante que una población puede exhibir.

El objetivo del presente artículo es analizar la relación estadística del reporte, en la encuesta antropométrica CANDELA, de tratamientos hormonales, cirugías maxilofaciales, ortodoncia, traumatismos y malformaciones (siguiendo la clasificación de Bishara et al., 2009) c, con los valores de asimetría fluctuante facial (AFF) en individuos mestizos latinoamericanos y caracterizar los cambios morfológicos asociados en aquellas variables respuesta que presenten diferencias estadísticamente significativas. La hipótesis nula del trabajo es que los valores de tendencia central y dispersión de la AFF no difieran entre grupos con condiciones diferentes de tratamientos hormonales, cirugías maxilofaciales, ortodoncia, traumatismos y malformaciones en individuos mestizos latinoamericanos, cuando estos sean sometidos a pruebas estadísticas.

\section{Sujetos y métodos}

\section{Voluntarios}

La muestra comprende un total de 2120 individuos femeninos (media de edad $=25.85$, d.e. $=9.24$ ) y 2004 masculinos (media de edad $=27.01$, d.e. $=9.31$ ) de entre 18 y 85 años, todos ellos participantes en el Consorcio para el Análisis de la Diversidad y Evolución de Latinoamérica-CANDELA (Ruiz-Linares et al., 2014).
Dentro del muestreo en CANDELA cada voluntario fue sometido a un proceso de: i) extracción de muestra sanguínea para su análisis genético, ii) medición y encuesta antropométricas, iii) toma de fotografías faciales y iv) cuestionario socioeconómico. Como parte del proceso de medición antropométrica a cada voluntario se le realizó un cuestionario denominado Hoja de Datos Fenotípicos, que consistió de siete medidas corporales, medición de la melanina, seis características fenotípicas como color de ojos, color de pelo, etc., y cuatro preguntas sobre elementos potenciales de modificación craneofacial: 1) malformaciones craneofaciales, 2) traumatismos faciales, 3) tratamientos hormonales y 4) tratamientos cirugías u ortodoncia craneofaciales. En dicho caso se respondía como "sí" o "no" y en algunos casos los voluntarios daban información del tipo de tratamiento, cirugía o malformación recibida, inclusive el tiempo del mismo, ello se ponía como información adicional (ver tabla suplementaria 1). Cabe destacar que no todos los voluntarios pudieron incluirse en los niveles de análisis (Tabla 1), debido a que en algunos casos los voluntarios no respondieron la encuesta CANDELA en su totalidad, por ello sólo se incluyen los casos que tenían la información completa en lo referente a las variables antes descritas. Luego entonces sobre estas variables se construyó un vector nominal para su posterior análisis. Dentro de las covariables que se preguntaron en el cuestionario socioeconómico CANDELA y que se han usado en el análisis están: sexo (dicotomizada como femenino [1] y masculino [2]), edad (considerada como variable continua), índice de masa corporal (IMC, considerada como variable continua), índice de melanina (variable continua) y heterocigosidad (porcentaje entre 0 y 1 ) resultado del análisis genómico de la ancestría (para mayor detalle ver Quinto-Sánchez et al., 2015, 2017).

\section{Estimación de la asimetría facial}

La caracterización de la asimetría facial se realizó por medio de un conjunto de 34 landmarks (ver Material Suplementario (MS) 1), 26 puntos bilaterales y 8 sagitales 
(para más detalles, ver Quinto-Sánchez et al., 2015, 2017), sobre una reconstrucción fotogramétrica del rostro realizada a través de las fotografías faciales tomadas a cada voluntario (ver MS1). La evaluación de la asimetría individual se realizó conforme a lo establecido por Klingenberg y McIntyre (1998) por medio del cálculo del Procrustes ANOVA y MANOVA para caracterizar el componente asimétrico de variación de la forma (shape) en el fenotipo facial (Klingenberg et al., 2002, 2010a). Estos autores sostienen que los organismos que exhiben simetría de objeto (object symmetry) necesitan un procedimiento especial para caracterizar las cantidades relativas de los componentes de variación de forma simétrica y asimétrica. Los análisis para las configuraciones con simetría de objeto, como lo es la cara, separan la configuración original de landmarks en dos componentes de variación: la simétrica y la asimétrica, usando un método de Procrustes por superposición de las configuraciones originales y sus imágenes especulares (Klingenberg et al., 2002). Ello permite obtener un valor (escalar) de asimetría fluctuante facial por individuo (AFF), que es el valor de ese individuo respecto a la media poblacional de la componente asimétrica de la forma (Mardia et al., 2000). El modelo ANOVA Procrustes estima la proporción de varianza de cada uno de los siguientes componentes: a) individual, b) un efecto lado, informativo de asimetría direccional, c) un efecto de interacción individuo por lado que indica la cantidad de asimetría fluctuante y además considera d) el efecto de error de medición (Klingenberg y McIntyre, 1998; Mardia et al., 2000). Para este trabajo se utilizó la estimación de AFF individual de Mahalanobis (Klingenberg y McIntyre, 1998; Klingenberg y Monteiro, 2005), la cual estandariza la variación por grupos. Teniendo en cuenta que la mayoría de las formas biológicas presentan una distribución de la variación no isotrópica a través de los landmarks (Klingenberg et al., 2002), también se realizó una prueba MANOVA que considera la naturaleza no isotrópica de las configuraciones de landmarks al estimar los efectos de asimetría direccional y fluctuante. Los valores $p$ se estimaron utilizando una permutación de la prueba basada en 100.000 iteraciones de los datos originales (ver MS2).

\section{Análisis de la relación estadística de la AFF y las variables respuesta}

Con el fin de evaluar las diferencias entre medias y varianzas de AFF a través de las diferentes variables respuesta se realizó un ANOVA de una vía. Asimismo, en los grupos cuyas varianzas resultaron significativamente diferentes, se aplicó la prueba de ANOVA de Welch. La estadística de Welch se basa en la prueba F ANOVA usual, pero las medias se ponderan por la inversa de la varianza media grupal (Welch, 1951). Al tener sólo dos niveles, como en el caso de este grupo de variables, la prueba de Welch es equivalente a una prueba $t$ de varianza desigual. En todas las pruebas se consideró un valor $\alpha=0.01$. Los análisis arriba descritos fueron repetidos sobre los residuales de la regresión multivariada (ver MS3) entre las covariables

TABLA 1. Tabulación cruzada del sexo y variables analizadas

\begin{tabular}{|c|c|c|c|c|c|}
\hline \multirow{2}{*}{$\frac{\text { Sexo }}{\text { Variable/condición }}$} & \multicolumn{2}{|c|}{ Mujer } & \multicolumn{2}{|c|}{ Hombre } & \multirow[b]{2}{*}{ Totales } \\
\hline & No & Sí & No & Sí & \\
\hline Malformación & 2094 & 26 & 1983 & 20 & 4123 \\
\hline Traumatismos & 2025 & 96 & 1795 & 209 & 4125 \\
\hline Tratamiento hormonal & 1842 & 275 & 1971 & 32 & 4120 \\
\hline Cirugía ortodoncia & 799 & 1321 & 821 & 1183 & 4124 \\
\hline
\end{tabular}


descritas arriba: sexo, edad, IMC, índice de melanina y heterocigosidad, indicador de ancestría genética ya reportado en trabajos previos (Quinto-Sánchez et al., 2015, 2017), ya que el modelo resultó en una asociación significativa $\left(\mathrm{F}=5.98, \quad p=0.0002, \mathrm{r}^{2}=0.12\right.$, RMSE $=1.193)$, específicamente con la heterocigosidad $(\mathrm{F}=19.19, p<0.001)$, lo anterior con la finalidad de evaluar si los resultados son persistentes una vez que las covariables antes descritas eran eliminadas del análisis mediante el uso de residuales (ver MS4 para detalles gráficos de la relación de las covariables y los valores de AFF).

En paralelo, para cada variable se graficó la proporción de densidades por sexo. Dichos gráficos comparan la distribución y composición de la AFF a través de las variables, mostrando la contribución de cada una en el modelo general.

Posteriormente, en aquellos grupos con significación estadística en el ANOVA de una vía, se exploró la interacción de la variable en cuestión con el resto para conocer si podría haber un efecto conjunto mediante un modelo de ANOVA anidado, que permite explorar los efectos cruzados de todas las interacciones posibles entre las cuatro variables en estudio.

Caracterización de la variación del componente asimétrico de la forma y varianza multidimensional por landmark

En aquellas variables que resultaron estadísticamente significativas en el ANOVA de una vía, se realizó un Análisis de Componentes Principales (ACP) a partir de matrices de varianza-covarianza del componente asimétrico de la forma. De este modo se caracterizó la máxima covariación entre los datos. Los ACP se calcularon para toda la muestra y para cada subgrupo respecto de las variables utilizadas (e.g. ingesta o no ingesta de hormonas), por lo que también se obtuvieron valores de varianza total y para cada variable. De igual manera, sobre cada matriz de covarianza generada, se calculó un Contraste de Matrices (Klingenberg, 2011) con el fin de conocer cuales matrices eran proporcionales y cuales iteraciones eran diferentes estadísticamente $(\alpha=0.01)$.

Aunado a esto se caracterizó la varianza multidimensional total del componente asimé- trico de la forma por landmark con el fin de caracterizar la varianza de los landmarks, por medio del cálculo de convex hull en tres dimensiones y varianza simple para cuando los puntos se encontraban sobre la norma lateral (ya que estos pierden un grado de libertad al alinearse en el ajuste de Procrustes y no se considera su varianza tridimensional).

Los cálculos de morfometría geométrica (MG) fueron realizados en el programa MorphoJ (Klingenberg, 2011), los análisis estadísticos en el programa JMP $9^{\circledR} \mathrm{y}$ el cálculo de la varianza multidimensional en Python por medio de un paquete pyhull (Barber et al., 1996).

\section{RESULTADOS}

\section{Relación estadística de los valores de AFF y el tratamiento hormonal}

Los análisis de ANOVA de una vía, Welsh y Levene (Tabla 2) muestran que existen diferencias por media y varianza en los valores de asimetría fluctuante facial para aquellas mujeres que estuvieron sometidas a algún tipo de tratamiento hormonal ( $p<0.001$, Fig. 1a), es decir, el grupo con tratamiento hormonal muestra mayor cantidad de asimetría, respecto del grupo sin tratamiento. El resto de las variables no mostró asociación, aun cuando se asumen varianzas desiguales (Tabla 2) y cabe destacar que dicho patrón continuó cuando se realizó el mismo cálculo sobre los residuales de la regresión multivariada. Todos los patrones observados en los gráficos de densidad resultaron más o menos homogéneos, empero en el caso de la gráfica del tratamiento hormonal (Fig. 1a) la mayor proporción de la muestra estuvo sobre los valores más altos de asimetría fluctuante.

\section{Variación del componente asimétrico y varianza multidimensional por landmark}

El CP1 colectó un 22.22\%, 22.00\% y $25.08 \%$ de la varianza del grupo en general, sin tratamiento y con tratamiento, respectivamente (ver MS5). El ACP de la muestra con el tratamiento hormonal no arrojó un patrón diferencial para los distintos casos que se incluían (Fig. 2a). Los cambios de asimetría asociados al 
TABLA 2. Resultados del ANOVA de una vía (a), prueba de Welsh (b) y prueba de Levene (b) para las variables utilizadas

a) ANOVA de una vía

\begin{tabular}{|c|c|c|c|c|c|}
\hline \multicolumn{6}{|c|}{ Mujeres } \\
\hline & $\mathrm{gl}$ & SS & MS & $\mathrm{F}$ & $\mathrm{p}$ \\
\hline Malformación & 1 & 0.10 & 0.10 & 0.081 & 0.775 \\
\hline Traumatismos & 1 & 0.72 & 0.72 & 0.558 & 0.455 \\
\hline Tratamiento hormonal & 1 & 65.52 & 65.52 & 52.268 & $<0.0001^{*}$ \\
\hline Cirugía ortodoncia & 1 & 3.31 & 3.31 & 2.583 & 0.108 \\
\hline \multicolumn{6}{|c|}{ Hombres } \\
\hline Malformación & 1 & 0.77 & 0.77 & 0.643 & 0.423 \\
\hline Traumatismos & 1 & 1.02 & 1.02 & 0.848 & 0.357 \\
\hline Tratamiento hormonal & 1 & 3.80 & 3.80 & 3.168 & 0.075 \\
\hline Cirugía ortodoncia & 1 & 2.99 & 2.99 & 2.489 & 0.115 \\
\hline
\end{tabular}

b) Prueba de Welsh

\begin{tabular}{lcccc}
\hline \multicolumn{5}{c}{ Mujeres } \\
\hline & gl & gl den & F & p \\
\hline Tratamiento hormonal & 1 & 328.57 & 42.330 & $<0.0001^{*}$ \\
\hline
\end{tabular}

c) Prueba de Levene

\begin{tabular}{lcccc}
\hline & gl & gl den & F & p \\
\hline Malformación & 1 & 2098 & 3.535 & 0.060 \\
Traumatismos & 1 & 2099 & 1.378 & 0.241 \\
Tratamiento hormonal & 1 & 2095 & 9.136 & 0.003 \\
Cirugía ortodoncia & 1 & 2098 & 0.685 & 0.408 \\
\hline & \multicolumn{4}{c}{} \\
\hline Malformación & 1 & 1976 & 0.163 & 0.687 \\
Traumatismos & 1 & 1977 & 0.318 & 0.573 \\
Tratamiento hormonal & 1 & 1976 & 0.000 & 0.988 \\
Cirugia ortodoncia & 1 & 1977 & 0.081 & 0.776 \\
\hline
\end{tabular}

*Significación persistente en iteración con residuales de las covariables en el modelo de regresión multivariada.

CP1 están relacionados con desplazamientos de landmarks en las orejas, nariz y boca, mientras que el $\mathrm{CP} 2$ explica variaciones en las orejas y el mentón (ver Fig. $2 b$ para detalles de dichos cambios). El CP3 expresa mayor variación de orejas y mentón (no mostrado). El contraste de matrices de covarianza por pares muestra las diferencias entre el grupo con tratamiento hormonal, que presenta mayor varianza dentro de la muestra, reduciéndose únicamente en el eigenvalor 26 (Fig. 2c).

La caracterización multivariada de la varianza de los landmarks por convex hull en el grupo con tratamiento hormonal, muestra el mismo patrón 

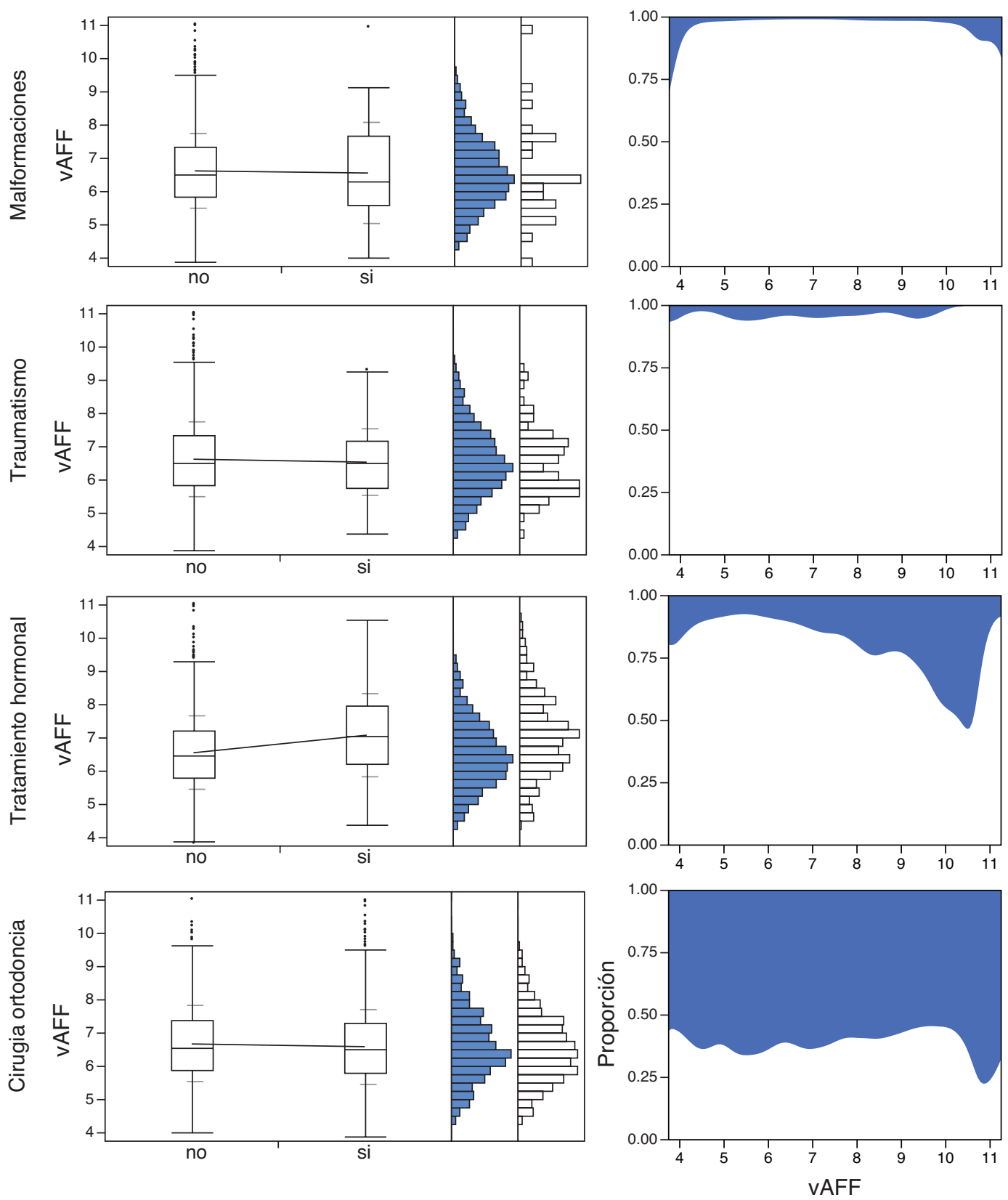

Fig. 1. Gráfica de cajas, histogramas de frecuencia y proporción de densidades para cada variable en mujeres en relación a los valores de AFF. Los círculos negros en las gráficas de cajas representan los individuos fuera del rango normal, la línea transversal entre las cajas conecta las medias y las líneas ortogonales a cada caja son las desviaciones estándar de la variable. El color azul representa el no y el blanco el sí.

que el ACP, es decir, una mayor varianza asociada a los puntos del mentón, maxilar, labio inferior, región perifrontal, región nasal y orejas (Fig. 3).

Entre las voluntarias que declararon consumir alguna medicación, únicamente se reportaron diferencias significativas en aquellas 275 que recibieron tratamiento hormonal. En este grupo de mujeres también se reportaron otras condiciones como cirugía maxilofacial $\mathrm{u}$ ortodoncia, sin embargo, no fueron observadas diferencias estadísticas asociadas por interacción con esta variable $(\mathrm{F}=3.24, \mathrm{p}=0.07)$, ni tampo- 

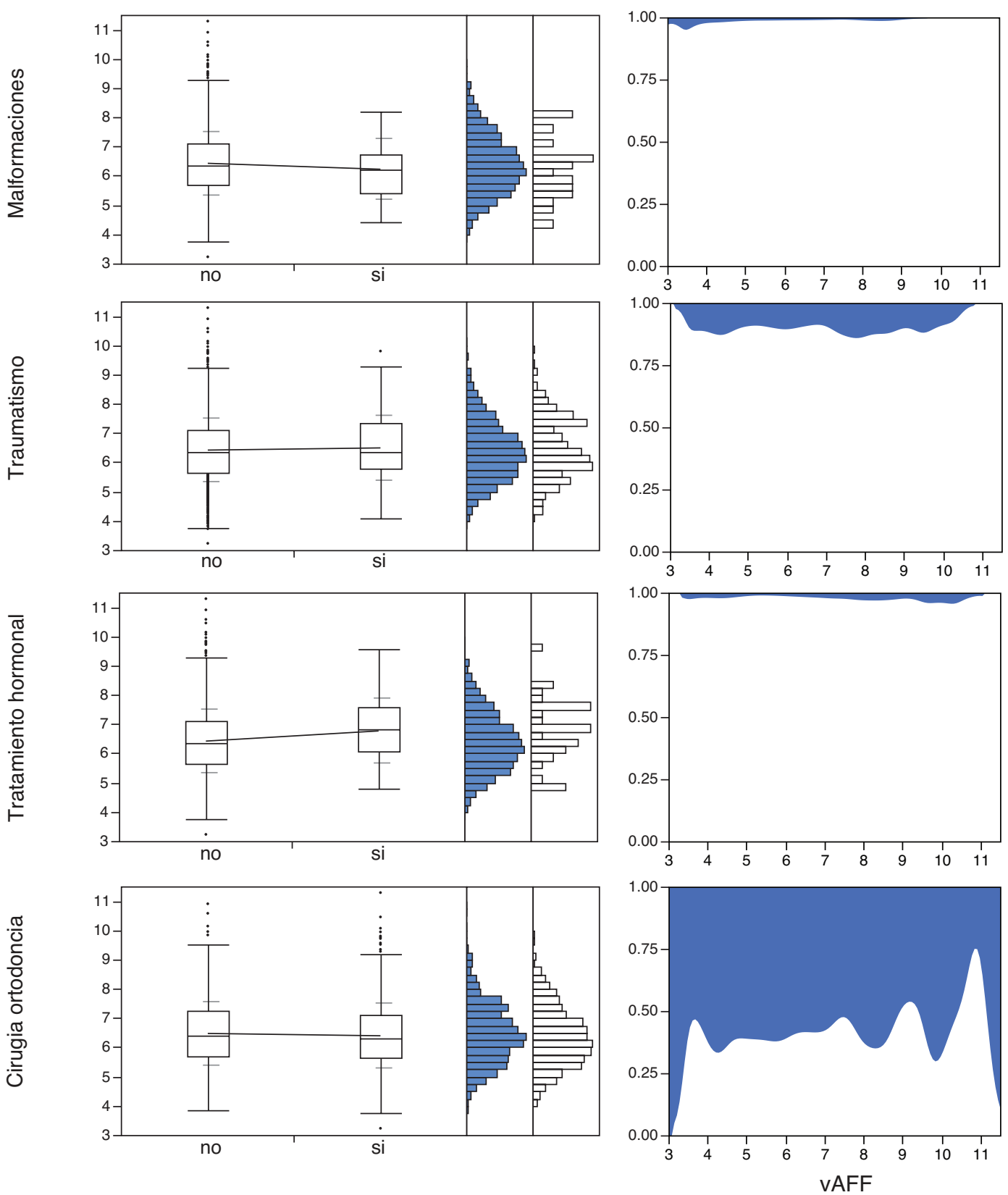

Fig. 1. Gráfica de cajas, histogramas de frecuencia y proporción de densidades para cada variable en hombres en relación a los valores de AFF. Los círculos negros en las gráficas de cajas representan los individuos fuera del rango normal, la línea transversal entre las cajas conecta las medias y las líneas ortogonales a cada caja son las desviaciones estándar de la variable. El color azul representa el no y el blanco el sí.

co con el resto de variables en toda la muestra $(\mathrm{F}=2.23, \mathrm{p}=0.13)$.

En la encuesta antropométrica realizada, las mujeres reportaron diversas causas del uso de tratamientos por hormonas. Estas fueron: "toma oral de anticonceptivos", "tratamiento con an- ticonceptivos por ovario poliquístico", "menopausia", "tratamiento para acné", "hipotiroidismo", "tratamiento con levotiroxina", "tiroides", "tratamiento para crecimiento", "anticonceptivos asociados a problemas en la menstruación” y "tratamiento con estrógeno". En general se 
documentaron diferentes diagnósticos en el consumo continuado de medicamentos: 241 casos estuvieron relacionados al consumo de hormonas, 4 al consumo de medicamentos por depresión y 2 de insulina. En el resto (7 casos) los voluntarios declararon tratamiento de neoplasias, control de colesterol, condición cardiaca, alergias, uso de vitaminas y enfermedades inflamatorias. En el caso del grupo de hombres, sólo 32 casos reportaron el consumo de algún tratamiento hormonal, empero estos no resultaron en diferencias significativas ( $\mathrm{p}=0.075$, ver Tabla 2 ).

\section{DISCUSIÓN}

El objetivo de este trabajo fue explorar el efecto de variables sugeridas previamente como potenciales modificadoras del fenotipo facial y su asimetría fluctuante, entendida como un indicador de inestabilidad en el desarrollo. La hipótesis nula fue rechazada $(\mathrm{p}<0.001)$ ya que una de las variables respuesta (el consumo de hormonas) mostró diferencias significativas, aun cuando el efecto de la ancestría genética (heterocigosidad) y otras covariables fue controlado estadísticamente en la muestra.

Respecto de las variables respuesta incluidas en el análisis existen evidencias claras de su posible efecto en el modelado craneofacial y por ende candidatas a estar relacionadas con la cantidad de AFF. En este sentido, DeLeon y Richtsmeier (2009) no encontraron evidencia del papel de la inestabilidad en el desarrollo del sistema etiológico de la craneosinostosis sagital no sindrómica, pero mostraron que existe relación localizada de la asimetría en los procesos clinoides anterior en el grupo de pacientes con sinostosis sagital, lo que sugiere una efecto de las extensiones de la duramadre que se adhieren allí. De igual manera, en una muestra de pacientes con síndrome de Down, se identificó que las prominencias faciales exhiben un aumento de asimetría fluctuante durante la morfogénesis del rostro, evidencia de una mayor inestabilidad en el desarrollo (Starbuck et al., 2013). Los autores reportan mayor inestabilidad en las estructuras faciales derivadas de la prominencia de la mandíbula y menor en las regiones faciales derivadas de la prominencia frontal (Starbuck et al., 2013). De igual manera, Klingenberg et al. (2010b) estudiando el efecto del consumo ma- terno de alcohol durante la gestación, encuentran que los grupos expuestos y no expuestos difieren significativamente, incrementándose la asimetría direccional promedio en los individuos del primer grupo. Basados en estos resultados, los autores concluyeron que la exposición al alcohol durante el desarrollo afecta a una amplia gama de características craneofaciales.

Entre las malformaciones congénitas se encuentran las maloclusiones y otros defectos en el desarrollo de la mandíbula. Se considera que la asimetría facial está presente aún en pacientes con excelente oclusión ("sanos o normales"), siendo la región lateral maxilar la que registra mayor cantidad de asimetría (Shah et al., 2009), existiendo además una tendencia direccional, donde el lado derecho está más afectado. En un estudio de la epidemiología de las oclusiones en poblaciones de EEUU realizado por Sheats et al. (1998) se mostró que entre los pacientes de ortodoncia, el rasgo asimétrico más frecuente fue la desviación de la línea media mandibular de la línea media facial ( $62 \%$ de los pacientes), seguido por la falta de coincidencia de la línea media dental (46\%), la desviación de la línea media del maxilar superior respecto de la línea media facial (39\%), la asimetría del molar (22\%), la asimetría oclusal maxilar (20\%), la asimetría mandibular oclusal (18\%), la asimetría facial (6\%), la desviación de la barbilla (4\%) y la desviación de la nariz (3\%).

En referencia a los traumatismos, en un estudio de pacientes de la India sobre incidencia de traumatismos faciales y lesiones craneales concomitantes (Rajendra et al., 2009), se describe que el hueso malar fue el hueso facial más comúnmente fracturado $(48.2 \%)$, seguido por fractura de mandíbula (42\%) y maxilar (39.4\%). $\mathrm{Al}$ repararse la lesión por traumatismo, es plausible que existan mayor cantidad de asimetrías (Markey et al., 1980).

El consumo de medicamentos también puede considerarse un factor ambiental de potencial interés para el estudio de las asimetrías. Se hace hincapié en los tratamientos hormonales ya que fue la única variable relacionada con la cantidad de asimetría en el rostro. Aunque hasta el momento no se encontró evidencia alguna sobre la relación directa entre los niveles de hormonas y las asimetrías faciales (Smith et al., 2006), una serie de estudios muestran el efecto que las 


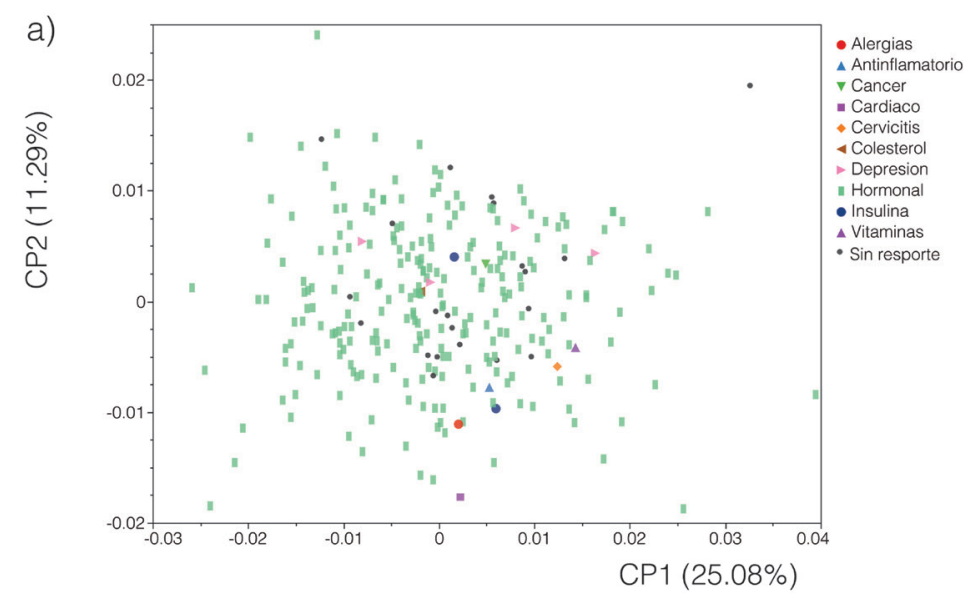

b)
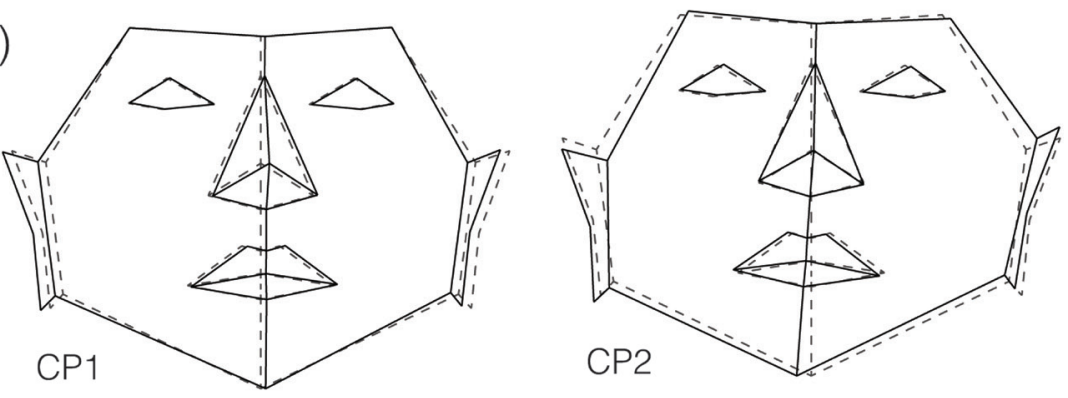

C)

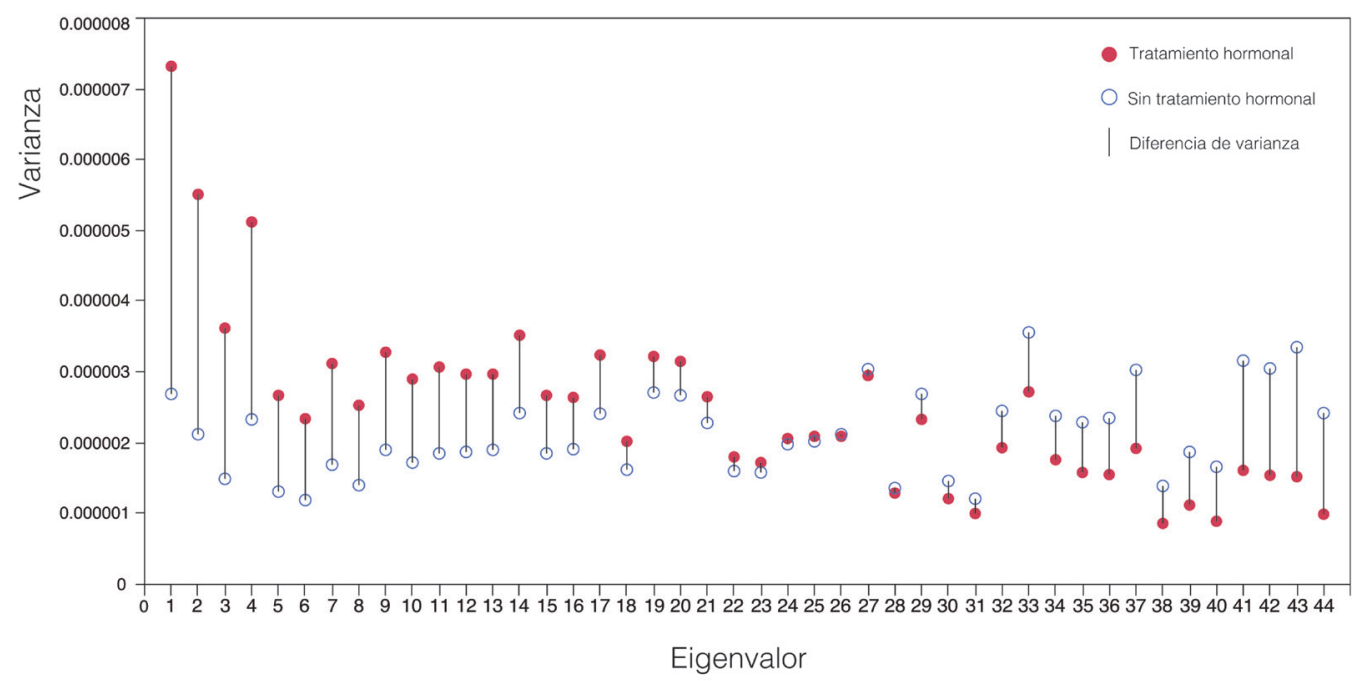

Fig. 2. Gráficos de dispersión (a), polígonos de deformación (b) y de contraste de varianza (c) de las matrices de covarianza del componente asimétrico. (a) CP1 y CP2 resultantes del ACP sobre la variación asimétrica de la forma, en diferentes colores y marcadores los tratamientos generales identificados por medio de la encuesta; (b) polígonos de deformación asociados al CP1 y CP2, la línea punteada representa la media y la línea continua la referencia de cambio para el extremo positivo del respectivo componente. Los vértices del polígono hacen referencia a los landmarks; (c) gráfica del contraste de varianza resultante del CM entre el grupo con tratamiento (círculo rojo) y sin tratamiento hormonal (círculo perímetro azul), las líneas en negro entre cada par de puntos representan la diferencia entre la varianza de ese componente en la matriz de covarianza. 


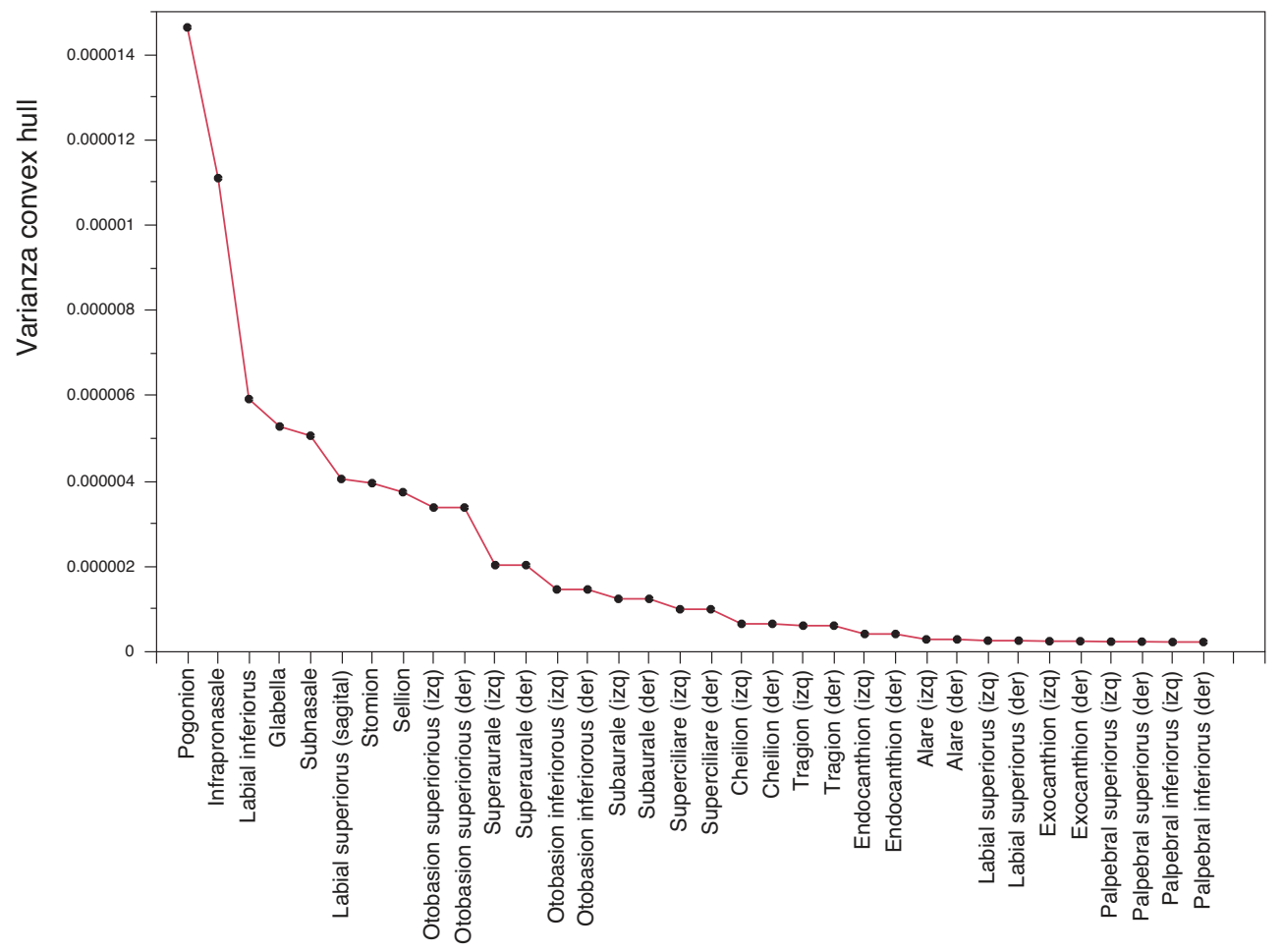

Fig. 3. Varianza observada en cada landmark, estimada por medio del cálculo de varianza multidimensional (convex hull). Los valores presentados de la máxima a la mínima variación.

hormonas tienen en la configuración del macizo craneofacial (Enlow, 1990, 1996; Kasperk et al., 1997; Neave, 2008). Inclusive, se ha especulado que las mujeres perciben cambios en su peso corporal durante la ingesta de tratamientos hormonales (Guendelman et al., 2000). De esta manera se combinan dos campos de evidencia indirecta del efecto de las hormonas en la estructura facial: la morfológica y la de la percepción corporal.

A nivel fisiológico, hay evidencia de que los receptores para andrógenos y estrógenos se encuentran en las células precursoras de la formación y modificación del tejido óseo (Kasperk et al., 1997), particularmente los andrógenos incrementan la proliferación y diferenciación de osteoblastos. Los estrógenos también incrementan la formación, diferenciación y proliferación osteoblástica (Neave, 2008). Los estrógenos, cuando hay ausencia de andrógenos, provocan un decrecimiento de la formación de osteoclastos (Neave, 2008). Este patrón fisiológico es el responsable de la elevada frecuen- cia de osteoporosis en mujeres menopaúsicas y postmenopáusicas (Neave, 2008). Además, dicha configuración fisiológica se ha asociado a rasgos o formas faciales más gráciles, como cejas más altas, una línea de la mandíbula pequeña y redondeada y unos labios más gruesos (Enlow, 1996).

El dimorfismo sexual, de hecho, se facilita ontogenéticamente por la relación de testosterona-estrógenos durante la adolescencia (Bardin y Catterall, 1981; Enlow, 1990; Swaddle y Reierson, 2002). En los hombres, esta influencia hormonal persiste hasta los 20 años (Enlow, 1990). Además se ha evidenciado que las concentraciones de hormonas pueden tener relación en el cambio a corto plazo en tejidos faciales (Manning, 2002).

El estrógeno, en combinación con la testosterona y las hormonas de crecimiento, interviene y promueve el crecimiento de los huesos faciales, al igual que en la estructura ósea del resto del cuerpo. También influye en el grosor de los labios (Thornhill y Gangestad, 1993; Thornhill 
y Grammer, 1999). Se ha registrado evidencia de alteración en la densidad mineral del hueso cuando se fluctúan los niveles de estrógeno (Berenson, 2001).

En estudios experimentales en ratas, Noda et al. (1994) establecieron que las inyecciones de esteroides anabólicos en hembras jóvenes aceleraron el crecimiento craneofacial. De igual manera, monitoreando el crecimiento facial después de una terapia suplementaria con testosterona, en un periodo de seis meses a un grupo de niños que estaban en la pubertad normal, se observó aceleramiento del crecimiento del hueso facial (Verdonck et al., 1999).

Investigaciones previas indican que algunas características en las mujeres cambian a lo largo de sus ciclos menstruales, por ejemplo: el tono de voz (Bryant y Haselton, 2009) o el color de la piel (van den Berghe y Frost, 1986). Otros estudios sugieren que los hombres pueden ser capaces de detectar estas señales en las mujeres, lo que llevaría a preferir determinados olores corporales (Doty et al., 1975; Singh y Bronstad, 2001; Kuukasjärvi et al., 2004 Havlicek et al., 2006; Gildersleeve et al., 2012), determinados tipos de rostros (Roberts et al., 2004) o voces (Pipitone y Gallup, 2008; Fischer et al., 2011; Nathan Pipitone y Gallup, 2012) Esta detección de señales reproductivas se da mayoritariamente durante la fase folicular tardía (fecundidad). El estradiol y la progesterona son posibles candidatos para mediar tales cambios (Kuukasjärvi et al., 2004; Bryant y Haselton, 2009).

Por otro lado, el uso de tratamientos anticonceptivos se ha asociado al cambio en el tono de voz (Amir et al., 2002; Amir y Kishon-Rabin, 2004) y algunas mujeres han reportado cambios en el peso corporal durante el tratamiento (Guendelman et al., 2000).

Como ya se ha destacado, en el presente trabajo las mujeres que resultaron con mayor cantidad de asimetría facial, también reportaron la ingesta de alguna hormona. El tipo de muestras y análisis empleados no permiten establecer unívocamente una relación causa-efecto entre ambos fenómenos. Sin embargo, los resultados representan una evidencia clara, en una muestra grande y con covariables controladas, de la posible relación entre algún tratamiento hormonal y la asimetría facial. Por lo que se considera que el patrón se asociación no se trata de una correlación espuria.

De acuerdo a los datos y su tratamiento estadístico se ha evidenciado que el consumo de hormonas es la variable de mayor peso en el modelo, aunque también es plausible que el modelo interactúe con otras variables quizás no evaluadas en este estudio debido a las limitantes propias del diseño experimental. En este sentido, los cambios asimétricos descritos aquí están por encima de los valores reportados para poblaciones "sanas" o "normales" (Ercan et al., 2008), lo que secunda la idea de la relación entre el consumo de hormonas y el patrón de asimetría fluctuante.

La estabilidad del desarrollo es la situación alcanzada cuando un organismo ha contenido adecuadamente las perturbaciones epigenéticas, mostrando su fenotipo un desarrollo programado (Markow, 1995). Cuando un organismo no ha solventado tales perturbaciones puede mostrar signos de inestabilidad, donde el origen de los disturbios puede ser genético, ambiental o el producto de una interacción genotipo-ambiente. En el caso en estudio, observamos que una vez eliminado el efecto de la ancestría genética de la muestra, los valores de significación para el tratamiento con hormonas continuaron siendo significativos. Por este motivo, se sugiere que la ingesta de hormonas operaría como un factor ambiental generador de fenotipos con mayor cantidad de asimetría facial. Estos resultados están en sintonía con aquellos estudios sobre exposición a niveles de alcohol durante la vida intrauterina (Klingenberg et al., 2010b), que reportan un aumento en la asimetría direccional promedio.

Finalmente, resta destacar que las técnicas de Procrustes ANOVA mediante el uso de la morfometría geométrica separa el componente del tamaño permitiendo un análisis no influenciado por este factor, lo que permite asegurar que varios de los problemas identificados por algunos autores en referencia a efecto del tamaño (van Dongen y Gangestad, 2011) sean resueltos, permitiendo desplazar las visiones morfométricas clásicas de la estimación de asimetría individual y asegurando que los datos no están sesgados o, por lo menos, son una mejor aproximación del efecto de las hormonas sobre el patrón facial asimétrico. 


\section{CONCLUSIONES}

Mediante el análisis de los valores individuales de asimetría fluctuante facial y algunas variables respuesta se pudo observar que las mujeres que recibieron algún tipo de tratamiento hormonal mostraron mayores valores de asimetría respecto del grupo que no tuvo tratamiento; además de que dicho efecto no interactúa estadísticamente con el resto de las variables analizadas, aun cuando el efecto de la heterocigosidad (ancestría genética) y otras covariables era controlado en el análisis. Los cambios asociados se concentran en el mentón, el maxilar, el labio inferior, la región perifrontal, la región nasal y las orejas y sustentan la noción de que la exposición a las hormonas desencadena una respuesta a nivel de tejidos óseos y blandos que redundan en un aumento de la asimetría facial.

\section{AGRADECIMIENTOS}

A los voluntarios del proyecto CANDELA, quienes hicieron posible la realización del estudio. A las entidades que financiaron el presente trabajo.

\section{LITERATURA CITADA}

Allenbach DM, Sullivan KB, Lydy MJ. 1999. Higher fluctuating asymmetry as a measure of susceptibility to pesticides in fishes. Environ Toxicol Chem 18:899-905. doi:10.1002/etc.5620180512

Amir O, Kishon-Rabin L. 2004. Association between birth control pills and voice quality. Laryngoscope 114:10211026. doi:10.1097/00005537-200406000-00012

Amir O, Kishon-Rabin L, Muchnik C. 2002. The effect of oral contraceptives on voice: preliminary observations. J Voice 16:267-273. doi:10.1016/S08921997(02)00096-6

Barber C, Dobkin D, Huhdanappa H. 1996. The quickhull algorithm for convex hulls. ACM Trans Math Softw 22:469-483. doi:10.1145/235815.235821

Bardin C, Catterall J. 1981. Testosterone: a major determinant of extragenital sexual dimorphism. Science (80- ) 211:1285-1294. doi:10.1126/science.7010603

Berenson A. 2001. A prospective, controlled study of the effects of hormonal contraception on bone mineral density. Obstet Gynecol 98:576-582. doi:10.1097/00006250200110000-00009

van den Berghe P, Frost P. 1986. Skin color preference, sexual dimorphism and sexual selection: a case of gene culture coevolution? Ethn Racial Stud 9:87-113. doi:10 $.1080 / 01419870.1986 .9993516$

Bishara SE, Burkey PS, Kharouf JG. 2009. Dental and facial asymmetries: a review. Angle Orthod 64:89-98.

Bjorksten T, David P, Pomiankowski A, Fowler K. 2000. Fluctuating asymmetry of sexual and nonsexual traits in stalk-eyed flies: a poor indicator of developmental stress and genetic quality. J Evol Biol 13:89-97. doi:10.1046/j.1420-9101.2000.00146.x

Bryant GA, Haselton MG. 2009. Vocal cues of ovulation in human females. Biol Lett 5:12-15. doi:10.1098/ rsbl.2008.0507

Burton C, Stevenson J, Williams D, Everson P, Mahoney E. 2002. Attention deficit disorder (ADD) and fluctuating asymmetry (FA) in a college sample. Am J Hum Biol 14(7). doi:10.1002/ajhb.20507

Claes P, Walters M, Shriver MD, Puts D, Gibson G, Clement J, Baynam G, Verbeke G, Vandermeulen D, Suetens P. 2012. Sexual dimorphism in multiple aspects of 3D facial symmetry and asymmetry defined by spatially dense geometric morphometrics. J Anat 221:97-114. doi:10.1111/j.1469-7580.2012.01528.x

Clarke GM. 1993. Fluctuating asymmetry of invertebrate populations as a biological indicator of environmental quality. Environ Pollut 82:207-211. doi:10.1016/02697491(93)90119-9

Debat V, David P. 2001. Mapping phenotypes: canalization, plasticity and developmental stability. Trends Ecol Evol 16:555-561. doi:10.1016/S0169-5347(01)02266-2

DeLeon VB, Richtsmeier JT. 2009. Fluctuating asymmetry and developmental instability in sagittal craniosynostosis. Cleft Palate Craniofac J 46:187-96. doi:10.1597/08001.1

van Dongen S, Gangestad SW. 2011. Human fluctuating asymmetry in relation to health and quality: a meta-analysis. Evol Hum Behav 32:380-398. Disponible en: http://linkinghub.elsevier.com/retrieve/ pii/S1090513811000249. doi:10.1016/j.evolhumbehav.2011.03.002

Doty RL, Ford M, Preti G, Huggins GR. 1975. Changes in the intensity and pleasantness of human vaginal odors during the menstrual cycle. Science 190:1316-1318. doi: $10.1126 /$ science. 1239080

Emlen JM, Freeman DC, Graham JH. 1993. Nonlinear growth dynamics and the origin of fluctuating asymmetry. Genetica 89:77-96. doi:10.1007/BF02424507

Enlow DH. 1990. Facial growth. Philadelphia: Saunders.

Enlow DH. 1996. Growth of the mandible. En: Enlow DH, Hans M, editores. Essentials of facial growth. Philadelphia: Saunders. p 57-78.

Ercan I, Ozdemir ST, Etoz A, Sigirli D, Tubbs RS, Loukas M, Guney I. 2008. Facial asymmetry in young healthy subjects evaluated by statistical shape analysis. J Anat 213:663-669. doi:10.1111/j.1469-7580.2008.01002.x

Farrera A. 2011. Evaluación de la asimetría facial mediante morfometría geométrica. Análisis de la simetría facial en una muestra de población mexicana. Tesis de pregrado en Antropología Física. Escuela Nacional de Antropología e Historia. INAH-SEP. México DF.

Farrera A, Villanueva M, Quinto-Sánchez M, González-José R. 2014. The relationship between facial shape asymmetry and attractiveness on Mexican students. Am J Hum Biol 27:387-396.

Fischer J, Semple S, Fickenscher G, Jürgens R, Kruse E, Heistermann M, Amir O. 2011. Do women's voices provide cues of the likelihood of ovulation? The importance of sampling regime. PLoS One 6:e24490. doi:10.1371/journal.pone.0024490

Gildersleeve KA, Haselton MG, Larson CM, Pillsworth EG. 2012. Body odor attractiveness as a cue of impending ovulation in women: evidence from a study using hormone-confirmed ovulation. Horm Behav 61:157-166. doi:10.1016/j.yhbeh.2011.11.005

Graham JH, Freeman DC, Emlen JM. 1993. Antisymmetry, 
directional asymmetry, and dynamic morphogenesis. Genetica 89:121-137. doi:10.1007/BF02424509

Gray PB, Marlowe F. 2009. Fluctuating asymmetry of a foraging population: the Hadza of Tanzania. Ann Hum Biol 29:495-501. doi:10.1080/03014460110112060

Guendelman S, Denny C, Mauldon J, Chetkovich C. 2000. Perceptions of hormonal contraceptive safety and side effects among low-income Latina and nonLatina women. Matern Child Health J 4:233-239. doi:10.1023/A:1026643621387

Havlicek J, Dvorakova R, Bartos L, Flegr J. 2006. Non-advertized does not mean concealed: body odour changes across the human menstrual cycle. Ethology 112:81-90. doi:10.1111/j.1439-0310.2006.01125.x

Kasperk C, Helmboldt A, Börcsök I, Heuthe S, Cloos O, Niethard F, Ziegler R. 1997. Skeletal site-dependent expression of the androgen receptor in human osteoblastic cell populations. Calcif Tissue Int 61:464-473. doi:10.1007/s002239900369

Klingenberg CP. 2011. MorphoJ: an integrated software package for geometric morphometrics. Mol Ecol Resour 11:353-357. doi:10.1111/j.1755-0998.2010.02924.x

Klingenberg CP, McIntyre GS. 1998. Geometric morphometrics of developmental instability: Analyzing patters of fluctuating asymmetry with Procrustes methods. Evolution (N Y) 52:1363-1375. doi:10.2307/2411306

Klingenberg CP, Monteiro LR. 2005. Distances and directions in multidimensional shape spaces: implications for morphometric applications. Syst Biol 54:678-688. doi:10.1080/10635150590947258

Klingenberg CP, Barluenga M, Meyer A. 2002. Shape analysis of symmetric structures: quantifying variation among individuals and asymmetry. Evolution (N Y) 56:1909-1920. doi:10.1111/j.0014-3820.2002. tb00117.x

Klingenberg CP, Debat V, Roff DA. 2010a. Quantitative genetics of shape in cricket wings: developmental integration in a functional structure. Evolution $(\mathrm{N} \mathrm{Y})$ 64:2935-2951. doi: 10.1111/j.1558-5646.2010.01030.x

Klingenberg CP, Wetherill L, Rogers J, Moore E, Ward R, Autti-Rämö I, Fagerlund A, Jacobson SW, Robinson LK, Hoyme HE, Mattson SN, Li TK, Riley EP, Foroud T. 2010b. Prenatal alcohol exposure alters the patterns of facial asymmetry. Alcohol 44:649-657. doi:10.1016/j.alcohol.2009.10.016

Kuukasjärvi S, Eriksson C, Koskela E, Mappes T, Nissinen K, Rantala M. 2004. Attractiveness of women's body odors over the menstrual cycle: the role of oral contraceptives and receiver sex. Behav Ecol 15:579-584. doi:10.1093/beheco/arh050

Leamy LJ, Klingenberg CP. 2005. The genetics and evolution of fluctuating asymmetry. Annu Rev Ecol Evol Syst 36:1-21. doi:10.1146/annurev.ecolsys.36.102003.152640

Leary RF, Allendorf FW. 1989. Fluctuating asymmetry as an indicator of stress: implications for conservation biology. Trends Ecol Evol 4:214-217. doi:10.1016/0169. 5347(89)90077-3

Lens L, van Dongen S. 2008. Fluctuating and directional asymmetry in natural bird populations exposed to different levels of habitat disturbance, as revealed by mixture analysis. Ecol Lett 3:516-522. doi:10.1046/j.14610248.2000.00181.x

Lens L, Van Dongen S, Kark S, Matthysen E. 2002. Fluctuating asymmetry as an indicator of fitness: can we bridge the gap between studies? Biol Rev Camb Philos Soc 77:27-38. doi:10.1017/S1464793101005796

Little AC, Jones BC, Waitt C, Tiddeman BP, Feinberg DR,
Perrett DI, Apicella CL, Marlowe FW. 2008. Symmetry is related to sexual dimorphism in faces: data across culture and species. PLoS One 3:e2106. https://doi. org/10.1371/journal.pone.0002106

Little AC, Paukner A, Woodward RA, Suomi SJ. 2012. Facial asymmetry is negatively related to condition in female macaque monkeys. Behav Ecol Sociobiol 66:1311-1318. doi:10.1007/s00265-012-1386-4

Manning J. 2002. Short-term changes in asymmetry and hormones in men. Evol Hum Behav 23:95-102. doi:10.1016/S1090-5138(01)00079-4

Manning JT, Scutt D, Whitehouse GH, Leinster SJ, Walton JM. 1996. Asymmetry and the menstrual cycle in women. Ethol Sociobiol 17:129-143. doi:10.1016/01623095(96)00001-5

Mardia K V, Bookstein FL, Moreton IJ. 2000. Statistical assessment of bilateral symmetry of shapes. Biometrika 87:285-300. doi:10.1093/biomet/87.2.285

Markey RJ, Potter BE, Moffett BC. 1980. Condylar trauma and facial asymmetry: An experimental study. J Maxillofac Surg 8:38-51. doi:10.1016/S0301-0503(80)80071-3

Markow T. 1995. Evolutionary ecology and development instability. Ann Rev Entomol 40:105-120. https://doi. org/10.1146/annurev.en.40.010195.000541

McKenzie JA, Clarke GM. 1988. Diazinon resistance, fluctuating asymmetry and fitness in the Australian sheep blowfly, Lucilia cuprina. Genetics 120:213-220.

Milne B, Belsky J, Poulton R, Thomson WM, Caspi A, Kieser J. 2003. Fluctuating asymmetry and physical health among young adults. Evol Hum Behav 24:53-63. doi:10.1016/S1090-5138(02)00120-4

Møller AP. 1990. Fluctuating asymmetry in male sexual ornaments may reliably reveal male quality. Anim Behav 40:1185-1187. doi:10.1016/S0003-3472(05)80187-3

Møller A. 1996. Development of fluctuating asymmetry in tail feathers of the barn swallow Hirundo rustica. J Evol Biol 9:677-694. doi:10.1046/j.14209101.1996.9060677.x

Møller A, Swaddle J. 1997. Asymmetry, developmental stability and evolution. Oxford: Oxford University Press.

Nathan Pipitone R, Gallup GG. 2012. The unique impact of menstruation on the female voice: implications for the evolution of menstrual cycle cues. Ethology 118:281291. doi:10.1111/j.1439-0310.2011.02010.x

Neave N. 2008. Hormones and behaviour: a psychological approach. Cambridge: Cambridge University Press.

Noda K, Chang HP, Takahashi I, Kinoshita Z, Kawamoto T. 1994. Effects of the anabolic steroid nandrolone phenylpropionate on craniofacial growth in rats. J Morphol 220:25-33. doi:10.1002/jmor.1052200104

Özener B. 2010a. Fluctuating and directional asymmetry in young human males: effect of heavy working condition and socioeconomic status. Am J Phys Anthropol 143:112-120. doi:10.1002/ajpa.21300

Özener B. 2010b. Brief communication: facial fluctuating asymmetry as a marker of sex differences of the response to phenotypic stresses. Am J Phys Anthropol 143:321-324. doi:10.1002/ajpa.21357

Özener B. 2011. Does urban poverty increase body fluctuating asymmetry? Coll Antropol 35:1001-1005.

Özener B, Fink B. 2010. Facial symmetry in young girls and boys from a slum and a control area of Ankara, Turkey. Evol Hum Behav 31:436-441. doi:10.1016/j.evolhumbehav.2010.06.003

Palmer AR, Strobeck C. 1986. Fluctuating asymmetry: measurement, analysis, patterns. Annu Rev Ecol Syst 17:391-421. doi:10.1146/annurev.es.17.110186.002135

Palmer A, Strobeck C, Chippindale A. 1994. Bilateral va- 
riation and the evolutionary origin of macroscopic asymmetries. En: Markow T, editor. Developmental instability: its origins and evolutionary implications. Tempe: Springer. p 203-220. doi:10.1007/978-94-0110830-0 15

Pipitone R⿳亠丷厂, Gallup GG. 2008. Women's voice attractiveness varies across the menstrual cycle. Evol Hum Behav 29:268-274. doi:10.1016/j.evolhumbehav.2008.02.001

Pound N, Lawson DW, Toma AM, Richmond S, Zhurov AI, Penton-Voak IS. 2014. Facial fluctuating asymmetry is not associated with childhood ill-health in a large British cohort study. Proc R Soc Lond B Biol Sci 281:e20141639. doi:10.1098/rspb.2014.1639

Quinto-Sánchez M, Adhikari K, Acuña-Alonzo V, Cintas C, Silva de Cerqueira CC, Ramallo V, Castillo L, Farrera A, Jaramillo C, Arias W, Fuentes M, Everardo P, de Avila F, Gomez-Valdés J, Hunemeier T, Gibbon S, Gallo C, Poletti G, Rosique J, Bortolini MC, CanizalesQuintero S, Rothhammer F, Bedoya G, Ruiz-Linares A, Gonzalez-José R. 2015. Facial asymmetry and genetic ancestry in Latin American admixed populations. Am J Phys Anthropol 157:58-70. doi:10.1002/ajpa.22688

Quinto-Sánchez M, Cintas C, Silva de Cerqueira CC, Ramallo V, Acuña-Alonzo V, Adhikari K, Castillo L, Gomez-Valdés J, Everardo P, De Avila F, Hünemeier T, Jaramillo C, Arias W, Fuentes M, Gallo C, Poletti G, Schuler-Faccini L, Bortolini MC, Canizales-Quinteros S, Rothhammer F, Bedoya G, Rosique J, Ruiz-Linares A, González-José R. 2017. Socioeconomic status is not related with facial fluctuating asymmetry: evidence from Latin-American populations. PLoS One 12:e0169287. doi:10.1371/journal.pone.0172418

Rajendra PB, Mathew TP, Agrawal A, Sabharawal G. 2009. Characteristics of associated craniofacial trauma in patients with head injuries: an experience with 100 cases. J Emerg Trauma Shock 2:89-94. doi:10.4103/09742700.50742

Roberts SC, Havlicek J, Flegr J, Hruskova M, Little AC, Jones BC, Perrett DI, Petrie M. 2004. Female facial attractiveness increases during the fertile phase of the menstrual cycle. Proc. R. Soc. Lond. B 271:S270-S272. doi:10.1098/rsbl.2004.0174

Ruiz-Linares A, Adhikari, Kaustubh Acuña-Alonzo V, Quinto-Sánchez M, Jaramillo C, Arias W, Fuentes M, Pizarro M, Everardo P, de Avila F, Gómez-Valdés J, León-Mimila P, Hunemeier T, Ramallo V, Silva de Cerqueira CC, Burley M-W, Konka E, Zagonel de Oliveira M, Veronez MR, Rubio-Codina M, Attanasio O, Gibbon S, Gallo C, Poletti G, Rosique J, Schuler-Faccini L, Salzano FM, Bortolini M-C, Canizales-Quinteros S, Rothhammer F, Bedoya G, Balding D, Gonzalez-José R. 2014. Admixture in Latin America: geographic structu- re, phenotipic diversity and self-perception of ancestry based on 7,342 individuals. PLoS Genet 10:e1004572. https://doi.org/10.1371/journal.pgen.1004572

Schlichting C, Pigliucci M. 1998. Phenotipic evolution. Sunderland: Sinauer Associates.

Shackelford TK, Larsen RJ. 1997. Facial asymmetry as an indicator of psychological, emotional, and physiological distress. J Pers Soc Psychol 72:456-466. doi:10.1037/0022-3514.72.2.456

Shah M, Sharad B, Joshi M. 2009. An assessment of asymmetry in the normal craniofacial complex. Angle Orthod 48:141-148.

Sheats RD, McGorray SE, Musmar Q, Wheeler TT, King GJ. 1998. Prevalence of orthodontic asymmetries. Semin Orthod 4:138-145. doi:10.1016/S1073-8746(98)80015-7

Singh D, Bronstad PM. 2001. Female body odour is a potential cue to ovulation. Proc Biol Sci 268:797-801. doi:10.1098/rspb.2001.1589

Smith MJL, Perrett DI, Jones BC, Cornwell RE, Moore FR, Feinberg DR, Boothroyd LG, Durrani SJ, Stirrat MR, Whiten S, Pitman RM, Hillier SG. 2006. Facial appearance is a cue to oestrogen levels in women. Proc Biol Sci 273:135-140. doi:10.1098/rspb.2005.3296

Soulé M. 1982. Allometric variation. 1. The theory and some consequences. Am Nat 120:751-764. doi: $10.1086 / 284028$

Starbuck JM, Cole TM, Reeves RH, Richtsmeier JT. 2013. Trisomy 21 and facial developmental instability. Am J Phys Anthropol 151:49-57. doi:10.1002/ ajpa. 22255

Swaddle JP, Reierson GW. 2002. Testosterone increases perceived dominance but not attractiveness in human males. Proc Biol Sci 269:2285-2289. doi:10.1098/ rspb.2002.2165

Thornhill R, Gangestad SW. 1993. Human facial beauty: averageness, symmetry, and parasite resistance. Hum Nat 4:237-269. doi:10.1007/BF02692201

Thornhill R, Grammer K. 1999. The body and face of woman. Evol Hum Behav 20:105-120. doi:10.1016/ S1090-5138(98)00044-0

Verdonck A, Gaethofs M, Carels C, de Zegher F. 1999. Effect of low-dose testosterone treatment on craniofacial growth in boys with delayed puberty. Eur J Orthod 21:137-143. doi:10.1093/ejo/21.2.137

Weisensee KE. 2013. Assessing the relationship between fluctuating asymmetry and cause of death in skeletal remains: a test of the developmental origins of health and disease hypothesis. Am J Hum Biol 25:411-417. doi:10.1002/ajhb.22390

Welch B. 1951. On the comparison of several mean values: an alternative approach. Biometrika 38:330-336. doi: $10.2307 / 2332579$ 